DOI:10.3233/NRE-171450

IOS Press

\title{
Top 25 NeuroRehabilitation articles
}

To all our readers,

NeuroRehabilitation is celebrating 25 years As part of our special 25th anniversary celebration, we have identified the 25 most cited articles ${ }^{1}$ published in our journal's history. As a special gift to readers, we have compiled a special online supplement containing these articles for your reading pleasure (http://www.iospress.nl/neurorehabilitation-celebrates-its-25th-anniversary/). Please enjoy and continue to rely on NeuroRehabilitation as your number one reference source for timely, relevant, thematic and empirical information on neurorehabililtation advances and practice.

With best wishes,

Nathan Zasler, M.D.

Jeffrey Kreutzer, Ph.D.

Co-Editors-in-Chief

\begin{tabular}{|c|c|c|c|c|c|c|}
\hline Cited by & Authors & Title & Year & Volume & Issue & $\overline{\text { Document Type }}$ \\
\hline 386 & $\begin{array}{l}\text { Hyder A.A., Wunderlich C.A., } \\
\text { Puvanachandra P., Gururaj G., } \\
\text { Kobusingye O.C. }\end{array}$ & $\begin{array}{l}\text { The impact of traumatic brain } \\
\text { injuries: A global perspective }\end{array}$ & 2007 & 22 & 5 & Review \\
\hline 161 & $\begin{array}{l}\text { Protas E.J., Mitchell K., Williams } \\
\text { A., Qureshy H., Caroline K., } \\
\text { Lai E.C. }\end{array}$ & $\begin{array}{l}\text { Gait and step training to reduce } \\
\text { falls in Parkinson's disease }\end{array}$ & 2005 & 20 & 3 & Article \\
\hline 135 & Toglia J., Kirk U. & $\begin{array}{l}\text { Understanding awareness deficits } \\
\text { following brain injury }\end{array}$ & 2000 & 15 & 1 & Article \\
\hline 124 & $\begin{array}{l}\text { Adamovich S.V., Fluet G.G., } \\
\text { Tunik E., Merians A.S. }\end{array}$ & $\begin{array}{l}\text { Sensorimotor training in virtual } \\
\text { reality: A review }\end{array}$ & 2009 & 25 & 1 & Review \\
\hline 120 & $\begin{array}{l}\text { McDonald B.C., Flashman L.A., } \\
\text { Saykin A.J. }\end{array}$ & $\begin{array}{l}\text { Executive dysfunction following } \\
\text { traumatic brain injury: Neural } \\
\text { substrates and treatment } \\
\text { strategies }\end{array}$ & 2002 & 17 & 4 & Review \\
\hline 96 & $\begin{array}{l}\text { Fazio V.C., Lovell M.R., } \\
\text { Pardini J.E., Collins M.W. }\end{array}$ & $\begin{array}{l}\text { The relation between } \\
\text { post-concussion symptoms and } \\
\text { neurocognitive performance in } \\
\text { concussed athletes }\end{array}$ & 2007 & 22 & 3 & Article \\
\hline 95 & Waller S.M., Whitall J. & $\begin{array}{l}\text { Bilateral arm training: Why and } \\
\text { who benefits? }\end{array}$ & 2008 & 23 & 1 & Review \\
\hline 89 & $\begin{array}{l}\text { Toole T., Hirsch M.A., Forkink } \\
\text { A., Lehman D.A., Maitland } \\
\text { C.G. }\end{array}$ & $\begin{array}{l}\text { The effects of a balance and } \\
\text { strength training program on } \\
\text { equilibrium in Parkinsonism: } \\
\text { A preliminary study }\end{array}$ & 2000 & 14 & 3 & Article \\
\hline 88 & $\begin{array}{l}\text { Haas C.T., Turbanski S., Kessler } \\
\text { K., Schmidtbleicher D. }\end{array}$ & $\begin{array}{l}\text { The effects of random } \\
\text { whole-body-vibration on motor } \\
\text { symptoms in Parkinson's } \\
\text { disease }\end{array}$ & 2006 & 21 & 1 & Article \\
\hline 82 & McAllister T.W., Arciniegas D. & $\begin{array}{l}\text { Evaluation and treatment of } \\
\text { post-concussive symptoms }\end{array}$ & 2002 & 17 & 4 & Review \\
\hline 79 & Clauss R., Nel W. & $\begin{array}{l}\text { Drug induced arousal from the } \\
\text { permanent vegetative state }\end{array}$ & 2006 & 21 & 1 & Article \\
\hline
\end{tabular}

\footnotetext{
${ }^{1}$ The table is a ranking of most cited articles published in NeuroRehabilitation (ISSN 1053-8135) between 1992 and 2017 , based on Scopus' citation data (Copyright @ 2017 Elsevier B.V.).
} 


\begin{tabular}{|c|c|c|c|c|c|c|}
\hline Cited by & Authors & Title & Year & Volume & Issue & $\overline{\text { Document Type }}$ \\
\hline 79 & $\begin{array}{l}\text { Morris D.M., Crago J.E., DeLuca } \\
\text { S.C., Pidikiti R.D., Taub E. }\end{array}$ & $\begin{array}{l}\text { Constraint-induced movement } \\
\text { therapy for motor recovery after } \\
\text { stroke }\end{array}$ & 1997 & 9 & 1 & Article \\
\hline 78 & $\begin{array}{l}\text { Krebs H.I., Mernoff S., Fasoli } \\
\text { S.E., Hughes R., Stein J., } \\
\text { Hogan N. }\end{array}$ & $\begin{array}{l}\text { A comparison of functional and } \\
\text { impairment-based robotic training } \\
\text { in severe to moderate chronic } \\
\text { stroke: A pilot study }\end{array}$ & 2008 & 23 & 1 & Article \\
\hline 77 & $\begin{array}{l}\text { Ptito A., Chen J.-K., } \\
\text { Johnston K.M. }\end{array}$ & $\begin{array}{l}\text { Contributions of functional Magnetic } \\
\text { Resonance Imaging (fMRI) to } \\
\text { sport concussion evaluation }\end{array}$ & 2007 & 22 & 3 & Article \\
\hline 75 & Busl K.M., Greer D.M. & $\begin{array}{l}\text { Hypoxic-ischemic brain injury: } \\
\text { Pathophysiology, neuropathology } \\
\text { and mechanisms }\end{array}$ & 2010 & 26 & 1 & Review \\
\hline 75 & Hesse $\mathrm{S}$. & $\begin{array}{l}\text { Treadmill training with partial body } \\
\text { weight support after stroke: A } \\
\text { review }\end{array}$ & 2008 & 23 & 1 & Review \\
\hline 75 & $\begin{array}{l}\text { Brown A.W., Leibson C.L., } \\
\text { Malec J.F., Perkins P.K., } \\
\text { Diehl N.N., Larson D.R. }\end{array}$ & $\begin{array}{l}\text { Long-term survival after traumatic } \\
\text { brain injury: A population-based } \\
\text { analysis }\end{array}$ & 2004 & 19 & 1 & Review \\
\hline 74 & Giacino J.T. & $\begin{array}{l}\text { The vegetative and minimally } \\
\text { conscious states: Consensus-based } \\
\text { criteria for establishing diagnosis } \\
\text { and prognosis }\end{array}$ & 2004 & 19 & 4 & Review \\
\hline 74 & $\begin{array}{l}\text { Harrison-Felix C., Whiteneck G., } \\
\text { DeVivo M., Hammond F.M., } \\
\text { Jha A. }\end{array}$ & $\begin{array}{l}\text { Mortality following rehabilitation in } \\
\text { the Traumatic Brain Injury Model } \\
\text { Systems of Care }\end{array}$ & 2004 & 19 & 1 & Article \\
\hline 72 & $\begin{array}{l}\text { Yasuda S., Wehman P., Targett P., } \\
\text { Cifu D.X., West M. }\end{array}$ & $\begin{array}{l}\text { Return to work after spinal cord } \\
\text { injury: A review of recent research }\end{array}$ & 2002 & 17 & 3 & Review \\
\hline 69 & Bedell G.M. & $\begin{array}{l}\text { Developing a follow-up survey } \\
\text { focused on participation of } \\
\text { children and youth with acquired } \\
\text { brain injuries after discharge from } \\
\text { inpatient rehabilitation }\end{array}$ & 2004 & 19 & 3 & Review \\
\hline 69 & $\begin{array}{l}\text { Bilbao A., Kennedy C., Chatterji } \\
\text { S., Üstün B., Barquero J.L.V., } \\
\text { Barth J.T. }\end{array}$ & $\begin{array}{l}\text { The ICF: Applications of the WHO } \\
\text { model of functioning, disability } \\
\text { and health to brain injury } \\
\text { rehabilitation }\end{array}$ & 2003 & 18 & 3 & Article \\
\hline 67 & $\begin{array}{l}\text { Strauss D., Ojdana K., Shavelle } \\
\text { R., Rosenbloom L. }\end{array}$ & $\begin{array}{l}\text { Decline in function and life } \\
\text { expectancy of older persons with } \\
\text { cerebral palsy }\end{array}$ & 2004 & 19 & 1 & Article \\
\hline 66 & $\begin{array}{l}\text { Tasiemski T., Kennedy P., } \\
\text { Gardner B.P., Taylor N. }\end{array}$ & $\begin{array}{l}\text { The association of sports and } \\
\text { physical recreation with life } \\
\text { satisfaction in a community sample } \\
\text { of people with spinal cord injuries }\end{array}$ & 2005 & 20 & 4 & Article \\
\hline 66 & $\begin{array}{l}\text { Nelson A.J., Zwick D., Brody S., } \\
\text { Doran C., Pulver L., Rooz G., } \\
\text { Sadownick M., Nelson R., } \\
\text { Rothman J. }\end{array}$ & $\begin{array}{l}\text { The validity of the GaitRite and the } \\
\text { functional ambulation performance } \\
\text { scoring system in the analysis of } \\
\text { Parkinson gait }\end{array}$ & 2002 & 17 & 3 & Article \\
\hline
\end{tabular}

